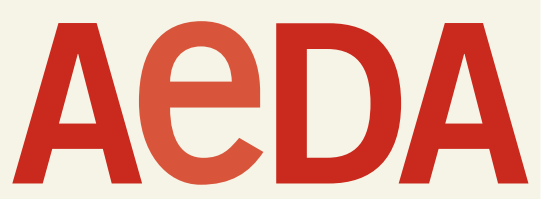

Ärzteverband Deutscher Allergologen (AeDA)

\section{Verantwortlich für den Inhalt}

Prof. Dr. Ludger Klimek

Zentrum für Rhinologie und Allergologie An den Quellen 10, 65183 Wiesbaden

Tel.: (06 11) 3086080

Fax: (06 11) 308608255

E-Mail: ludger.klimek@allergiezentrum.org

\section{AeDA-Geschäftsstelle}

Service Systems

Blumenstraße 14, 63303 Dreieich

Tel.: (0 61 03) 62273

Fax: (0 61 03) 697019

E-Mail: info@aeda.de

www.aeda.de

\section{Aluminium in Subkutanpräparaten}

\section{Alte Story, neu aufgewärmt}

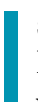
st damit zur rechnen, dass das Paul-Ehrlich-Institut (PEI) künftig eine Neubewertung der Aluminiumtoxikologie bei SIT-Lösungen vornehmen wird? Diese Frage stellte der Ärzteverband Deutscher Allergologen (AeDA) in seinem Schreiben vom 13. August an das PEI. Der AeDA bezog sich dabei auf die durch die European Food Safety Authority (EFSA) festgelegte Belastungstoleranzgrenze von $8,6 \mathrm{mg}$ Aluminium pro Tag, von der jüngst z.B. im Deutschen Ärzteblatt zu lesen war.

In der Antwort, die Vizepräsident Prof. Dr. Stefan Vieths am 29. August an den AeDA geschickt hat, stellt er zunächst klar, dass dieser von der EFSA festgelegte Wert sich aktuell nicht geändert hat: „Vielmehr scheint der Wert von $8,6 \mathrm{mg} / \mathrm{Tag}$ eine Umrechnung aus dem EFSA-Toleranzwert für einen $60 \mathrm{~kg}$ schweren Erwachsenen zu sein“, so Vieths. Der TWI-Wert (,tolerable weekly intake"), auf den sich Vieths hier bezieht, sei „nicht direkt auf die Exposition durch die subkutane Behandlung mit Therapieallergenen anwendbar, stelle aber dennoch eine gewisse Richtgröße dar".

Deutsche Gesellschaft für Allergolog und klinische Immunologie (DGAKI)

Verantwortlich für die Inhalte

Prof. Dr. Thilo Jakob

Allergieabteilung und Forschergruppe

Allergologie, Universitäts-Hautklinik

Freiburg

Hauptstraße 7, 79104 Freiburg

Tel.: (07 61) 2706714

Fax: (07 61) 2706655

E-Mail: thilo.jakob@uniklinik-freiburg.de

\section{DGAKI-Geschäftsstelle}

Gertrud Hammel

Schloss 2, 86482 Aystetten

Tel.: (08 21) 48687864

Fax: (08 21) 48687863

E-Mail:info@dgaki.de

www.dgaki.de

\section{Neubewertung ungewiss}

Unabhängig davon, erläutert Vieths, findet derzeit aufgrund der allgemeinen Diskussionen um die Sicherheit von Aluminium als Adjuvans eine Überprüfung der Präparate zur subkutanen Immuntherapie bzgl. der Aluminiumexposition statt. Zurzeit werden in einigen klinischen Studien Daten zur Aluminiumexposition erhoben. Die ersten Ergebnisse werden noch für dieses Jahr erwartet. Es ist jedoch unklar, ob dies zu einer Neubewertung der Aluminiumtoxikologie bei SIT-Lösungen führen wird. Im Antwortschreiben des PEI heißt es: „Die Analyse dieser Daten aus mehreren Studien wird zu einer aktu-

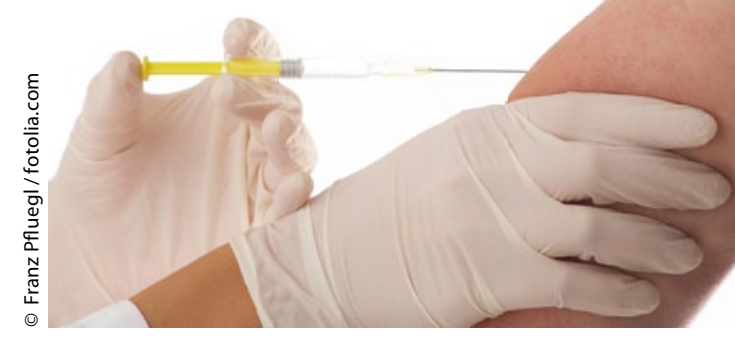

ellen Bewertung der Sicherheit von Aluminium in Präparaten zur SIT führen. Hierbei wird auch der Toleranzwert der EFSA Berücksichtigung finden. Inwieweit diese Analyse zu regulatorischen Änderungen führt, kann zurzeit nicht beurteilt werden.“

\section{Patienten aufklären}

Zudem wollte der AeDA wissen, ob das PEI Ärzten, die eine SIT durchführen, empfiehlt, auf mögliche unerwünschte Wirkungen des Aluminiums hinzuweisen und welche (Langzeit-)Wirkungen benannt werden sollten. Die Antwort des PEI lautet: „Generell müssen die behandelnden Ärzte über Nutzen und bekannte Risiken der Therapie aufklären, wobei sie jedoch nicht verpflichtet sind, über einzelne Bestandteile des Arzneimittels aufzuklären. Bekannte Risiken im Zusammenhang mit dem Aluminiumadjuvans in Allergenpräparaten sind Beschwerden/Fremdkörperreaktionen (Knötchenbildung) an der Injektionsstelle, über die insofern auf jeden Fall aufgeklärt werden sollte." Das Bundesgesundheitsblatt [Bundesgesundheitsbl 2009; 52: 1053-64] nimmt zur Problematik wie folgt Stellung: „Bisher gibt es keine wissenschaftliche Grundlage, die erklärt, wie eine akute Neurotoxizität aufgrund hoher Aluminiumkonzentrationen mit chronischen Krankheitsbildern im Zusammenhang stehen könnte.“

PD Dr. Kirsten Jung, Prof. Dr. Ludger Klimek 